\title{
NATURAL DIAMONDS GROWTH CONDITIONS ACCORDING TO THE MINERAL INCLUSIONS STUDY.
}

\author{
Bulanova, G.P. \\ Yakutian Institute of Geological Sciences.
}

The method of diamond cutting and polishing we used allows to study syngenitic inclusions in diamonds "in situ". The inclusions investigation is provided in close connection with diamond internal structure and its physical properties. The new approach to the diamond genesis reconstruction is fouded on the idea of 3 heterogeneous areas existence in diamond crystals: central (yellow luminescence), intermediate (blue luminescence) and periferal one (no luminescence) (Bescrovanov, 1986). Accordingly, in diamond growth history there were three stages differing in physical-chemical conditions. The above areas particularly well seen if the growth form change is observed in crystal: cube (cubo-octahedral or rounded form) octahedren. This sequence of areas in diamonds reflectes the main line of diamond growth evolution, sometimes it may differ.

Inclusion confinement to this quazihomogeneous on physical properties area permit comparing of the chemical alteration in inclusions of the same name both in one diamond and in different ones. Thus it makes possible to identify the evolution of environment chemistry and PT-regime in diamond genesis. Only such confinement or location we consider to be genetic. Accordingly, only those syngenetic inclusions which belong to the same area of diamond are considered to be in true mineral equilibrium. Because the above zonal structure is thin it is evident that dramatic alteration in inclusion chemistry is unlike. We identified the differences in olivine and chromite composition. For inclusions of eclogite association they are fixed in the composition of omphocite and pyrope-almandine. Besides, the order of ultrabasic silicate inclusions crystallisation for the time of diamond growth was established: $\mathrm{Ol}->\mathrm{Ol}+\mathrm{En}+\mathrm{Gr}->\mathrm{Ol}+\mathrm{En}+\mathrm{Gr}+\mathrm{Cpx}$. This order is intirely the same as the silicate crystallization order in ultrabasic melt (fluid). Thefore one can conclude that these diamonds grow as the first phase from carbon solution in silicate melt.

Our investigation didn't show any difference in internal structure of diamonds with ultrabasic and eclogitic inclusions suite. So we conclude that these diamonds grew in liquid environment too.

Besides zonal structure octahedren diamonds have zonal-sectoral and sectoral ones. It seems that macroinclusions (more than $20 \mathrm{mk}$ ) are located only in octahedren pyramids of grouth in suth diamonds. Microinclusions (less than $20 \mathrm{mk}$ ) and particules were located in its cubic growth pyramides only.

It is a vary rare event when octahedren diamonds have no zonal structure. Our study shows that inclusions in them have no differences in composition.

Lately we began to study such morphological varieties of diamond as cubes and coated diamonds. Coated diamonds represent in contrast with thin zonality the rough one. The identity of the coat material and cubic diamonds (the II-Orlo's variety) is confirmed by their physical properties. It seems that cubic diamonds contain only microinclusions, like those in cubic sectors of octahedren diamonds. We identified rutile, sanidine, apatite, mica and carbonate microinclusions in cubic diamonds. Similar in composition microinclusions were founded in coated diamonds. They are presented by mixture of sanidine, rutile, apatite and 
coesite (+/-Bt). Unfortunately, we failed to identify exactly their nature. Are they melt, partly crystalline or crystalline ones still unknown. But what is vary important is the fact that they belong to eclogite association.

Within the ontogenetic approack to diamond investigation we began together with Matsyuk and Platonov (IGFM, Kiev) to study optical spectra of Gr, Ol, Cpx, and chromite. The re ' of the investigation shwws that these minerals from diamonds have some crystalchemical features which distinguish them from the minerals of mantle xenoliths. The main distinction is the lowest concentration of optically active centres of $\mathrm{Fe}^{3+}$ ions in mineral inclusions. It indicates a very low redox condition of their crystallization. The presure of $\mathrm{Cr}^{2+}$ ions in spinels confirm such conclusions. The ions of $\mathrm{Cr}^{3+}$ are in other mineral structures, including olivine. In enstatite structure $\mathrm{Cr}$ enters according jadeite izomorph scheme as Sobolev believed earlier. We plan to extend these investigations for inclusions from different areas of the same diamond crystal.

We consider that the ontogenetic approach to diamond and its syngenetic inclusions study is the most up-to-date and perspective method of investigation. Using it we hope to create diamond genesis model, based on the most objective avaliable information given by diamonds themselves. 